Nel contesto del capitalismo delle piattaforme, un ruolo centrale viene occupato dal settore del food delivery che, spesso, in Italia e in Europa, è stato terreno di conflitto tra riders e aziende del settore. A essere oggetto di contesa sono quelle caratteristiche che vengono associate alla gig economy: fuga dai diritti del lavoro subordinato, estrema flessibilità, ricorso al pagamento a cottimo, mancanza di possibilità di esercitare i diritti associativi e collettivi (Friedman, 2014; Prassl, 2018).

La tendenza da parte delle piattaforme a eludere gli effetti della subordinazione, importando all'interno di contesti formalmente regolati le stesse caratteristiche di povertà e insicurezza che caratterizzano il lavoro informale (De Stefano, 2016), ha però un impatto anche sui processi organizzativi dei fattorini. A spingere $\mathrm{i}$ fattorini ad associarsi fuori dagli spazi sindacali tradizionali, dunque, è proprio tale condizione, che finisce con il privare i riders non solo dei diritti riconosciuti alla condizione di lavoratore subordinato, ma anche della possibilità di rivendicarli attraverso gli strumenti tradizionali della rappresentanza, più che un antagonismo nei confronti dei sindacati confederali. Questa forma dell'azione sindacale caratterizza da tempo lo scenario economico globale, dove il lavoro informale continua ad aumentare non solo in quei contesti dove è storicamente presente - come ad esempio il subcontinente indiano - ma anche in occidente, dove il trentennio di controriforme neoliberali è riu-

* Nicola Quondamatteo: Riders Union Bologna. Marco Marrone: Dipartimento di Managent, Università Ca’ Foscari di Venezia. 
scito a minare gli standard di tutela sul lavoro (Breman, van der Linden, 2015). Il capitalismo digitale, da questo punto di vista, rappresenta solamente una delle lance più affilate di tale processo, che non solo punta alla disintermediazione del rapporto di lavoro, ma finisce anche con il tradursi in una svolta autoritaria nell'ambito delle relazioni industriali (Scholz, 2017).

In Italia, come nel resto d'Europa, il mercato delle consegne di cibo a domicilio è in forte crescita (Di Vico, 2017), trainato dalle piattaforme che, grazie all'impiego delle tecnologie digitali, sono arrivate a trasformare un servizio marginale, storicamente condotto nell'informalità, in un business in grado di registrare tassi di crescita esponenziali. Un'esplosione che però porta con sé anche una particolare dinamicità, in cui è sempre più protagonista un oligopolio di multinazionali (Deliveroo, Just Eat, Uber Eats, Glovo, Foodora) che sta rapidamente espellendo le numerose start-up italiane. Inoltre, sono in corso processi di fusione e concentrazione, sia nel caso delle aziende locali sia per quanto riguarda le imprese maggiori: Glovo, ad esempio, ha rilevato le attività italiane di Foodora.

Nonostante tale crescita però, i contratti utilizzati continuano a essere però i co.co.co o le prestazioni occasionali (Cavallini, 2017). Mancano tutele base sulla sicurezza, sulle ferie, sulla malattia; sul fronte salariale le aziende possono unilateralmente cambiare quantità e modalità della paga, esprimendo una chiara tendenza a retribuire a cottimo i propri lavoratori. In questo contesto avvengono le esperienze associative dei lavoratori; interessante - da questo punto di vista - è analizzare l'esperienza bolognese di Riders Union che non solo è stata in grado di attirare l'attenzione mediatica, ma è anche riuscita a dar vita, con la firma della Carta dei diritti dei lavoratori digitali, avvenuta a Bologna nel maggio del 2018, al primo accordo europeo tra piattaforme digitali e rappresentanze informali dei lavoratori.

\section{L'esperienza di Riders Union}

Prima ancora di analizzare le peculiarità organizzative del sindacato informale dei riders, è interessante sottolineare alcuni fenomeni "sottotraccia" che costituiscono un retroterra prezioso per comprendere le ragioni di tali scelte. La natura urbana di questo lavoro consente infatti ai riders di socializzare durante l'attesa dell'assegnazione degli ordini, che diventa un momento peculiare per la concentrazione nello stesso luogo di una forza lavoro facilmente riconoscibile grazie al branding imposto da ciascuna azienda e che finisce per rovesciarsi in elemento simbolico e identitario ben preciso (es. il cubo in spalla). Questo è un elemento deci- 
sivo per spiegare la peculiarità dei riders nel contesto della gig economy perché costituisce un elemento di controtendenza rispetto ai processi di frammentazione e individualizzazione della prestazione lavorativa. Stesso discorso si può fare per la nascita dei gruppi Whatsapp e Telegram tra colleghi, anch'essi frutto di un rovesciamento delle strategie aziendali di governo della forza lavoro, che vedono l'impiego di gruppi Whatsapp e Telegram come strumenti per riuscire a coordinare la propria flotta. Così, già prima della nascita di Riders Union Bologna (Rub), si assiste al moltiplicarsi di fianco alle chat aziendali, gestite e supervisionate dai dispatcher, di gruppi organizzati dai lavoratori. (Marrone, 2019).

Il primo vero e proprio nucleo organizzativo dei riders bolognesi nasce da una manciata di fattorini di Deliveroo desiderosi di incontrarsi per discutere le problematiche legate alla loro condizione. Trovano così ospitalità presso il circolo Arci Ritmolento, uno spazio sociale già attivo su questi temi e che aveva sviluppato due iniziative: una campagna sociale e comunicativa contro la precarietà e uno sportello di orientamento al lavoro rivolto a coloro che sono impegnati nella ricerca della prima occupazione. In questa prima riunione si parla dei rischi di impresa totalmente scaricati sui lavoratori: in particolare l'attenzione viene posta sui costi di manutenzione del mezzo. Viene deciso così di attivare una ciclo-officina per autoriparazioni di base (es. il cambio della camera d'aria); iniziano poi passaparola e volantinaggi tra i colleghi, dando così forma a un primo nucleo organizzativo.

Un'accelerazione viene data dallo sciopero spontaneo del 13 novembre 2017, in cui, a seguito di un'improvvisa nevicata che aveva reso impraticabili le strade di Bologna, i lavoratori di tutte le piattaforme si rifiutano di svolgere il servizio. A seguito di tale iniziativa viene così palesata l'attività del sindacato informale - che fino ad ora aveva operato stando attento a non esporre i propri membri per evitare che venissero disconnessi come già successo a Torino in occasione del primo sciopero organizzato dai lavoratori di Foodora - con un post Facebook che supera le 4.000 condivisioni, finendo così con l'attirare l'attenzione dei maggiori quotidiani locali. Un successo più che altro simbolico, dato che lo sciopero non riesce a bloccare il servizio di consegna, ma riesce comunque ad aprire una finestra mediatica entro cui tentare di costruire radicamento tra i lavoratori. A questo proposito, un ruolo decisivo viene giocato dal mutualismo: la ciclo-officina e le bici di sostituzione (che i riders possono prendere temporaneamente in prestito in attesa della riparazione del proprio mezzo) rispondono a bisogno immediati e concreti che i lavoratori sentono e che le aziende non coprono. Come disse un rider durante un'assemblea «Riders Union non è solo una realtà politica, è anche una 
realtà pragmatica». In un contesto di lavoro fortemente precarizzato e informalizzato tornano quindi d'attualità le pratiche di mutuo aiuto che furono alla base della nascita del movimento operaio europeo (Cannavò, 2018). Allo stesso modo, un'esperienza importante per socializzare e rompere l'individualismo e l'atomizzazione tipici della gig economy sono i dopolavoro, che spesso accompagnano le riunioni e le assemblee e che rappresentano i luoghi di condivisione della quotidianità lavorativa.

Nell'elaborazione delle proprie rivendicazioni, i lavoratori - pur non sentendosi affatto autonomi e "imprenditori di sé stessi" - non partono mai dalla tipologia di inquadramento contrattuale, ma dalla serie di diritti di base, propri del lavoro subordinato, che le piattaforme eludono. Uno su tutti è quello della sicurezza e della mancanza di una copertura assicurativa. «Se cado e mi rompo un braccio, non solo non ho nessuna garanzia ma non posso nemmeno portare avanti l'attività musicale, che è l'altra mia fonte di reddito insieme e Deliveroo» - dice un rider in un'assemblea dell'autunno 2017. Prima ancora che la componente salariale, a ricoprire un ruolo centrale nelle rivendicazioni elaborate da Rub è la questione della sicurezza e l'assenza di una copertura assicurativa. Si tratta di una problematica amplificata da un modello organizzativo, basato su un largo impiego del controllo digitale e su una costante sollecitazione all'intensificazione della prestazione determinata dal cottimo, che finisce non solo con lo scaricare sulle spalle dei lavoratori i costi di tali rischi, ma anche a incrementare esponenzialmente i rischi stessi. Importante poi è la richiesta di un monte ore garantito; come per l'esperienza dei portuali (Marrone, 2019), riuscire a garantire un minimo di ore individuale è uno strumento essenziale per riuscire a prevenire le pratiche di "assunzioni selvagge", che vedono le piattaforme sostituire periodicamente larga parte della propria flotta proprio per evitare il formarsi di possibili aggregati conflittuali.

Per poter vedere realizzate tali richieste, però, a causa della difficoltà nel riuscire a condizionare i comportamenti economici delle piattaforme, si è deciso di far leva sull'opinione pubblica, sollecitata attraverso l'utilizzo dei social network, ma anche di pratiche tipiche dei movimenti sociali, come presidi e flash mob in luoghi del centro cittadino. In uno di questi, il 24 novembre 2017, i lavoratori si presentano sotto le due torri mascherati da ciclisti: questo sia per proteggere l'identità in un contesto di rapporti di forza sfavorevoli e di assenza di tutela sindacale e contro il licenziamento, sia per ribaltare la narrazione delle piattaforme secondo cui consegnare pizze non è un lavoro, ma un lavoretto o un hobby.

Una volta ottenuto l'interesse dei media locali e nazionali, i riders provano poi a chiamare in causa le istituzioni per riuscire a incrementare 
la pressione nei confronti delle piattaforme. Ciò avviene a partire dalle istituzioni locali, chiamando in causa con una lettera il Sindaco che viene invitato a attivarsi in quanto responsabile amministrativo del luogo in cui avviene il "processo produttivo" delle piattaforme. In particolare, come accaduto già negli Usa e, in maniera crescente, anche in diverse città europee dove l'esplosione delle piattaforme ha generato un impatto su questioni decisive come quelle degli affitti o del lavoro di cura, Rub sollecita l'intervento a protezione non solo dei lavoratori del food delivery, ma dell'intera città minacciata dagli atteggiamenti estrattivi delle piattaforme (Comito, 2018).

È a seguito di queste sollecitazioni che prende forma un tavolo di confronto con il Comune di Bologna dal quale originerà la Carta dei diritti fondamentali del lavoro digitale nel contesto urbano. Si tratta di uno strumento che anche se non è in grado di incidere sulla questione centrale della qualificazione del rapporto di lavoro dei riders, riesce a riconoscere un livello minimo di tutele (salario orario legato ai Ccnl, assicurazione, indennità per festivi ecc.) vincolanti a coloro che la sottoscrivono a titolo volontario. La Carta è inizialmente sottoscritta dalla sola Sgnam, azienda locale operante sul territorio di Bologna, per poi vedere anche la firma di Domino's Pizza, ma non dalle multinazionali del settore, che si sono invece rifiutate di aderire all'iniziativa promossa dall'amministrazione comunale. In ogni caso, essa rappresenta un'arma fondamentale nelle mani di Rub non solo per i diritti che vengono riconosciuti a una parte significativa dei lavoratori operanti sul terreno di Bologna, ma anche perché consentono di mobilitare un capitale simbolico in grado di sollecitare l'interesse del Governo che, pochi giorni dopo la firma della Carta, convoca le rappresentanze dei riders nel primo incontro ufficiale del nuovo Ministro del Lavoro. Questo si è concluso con un nulla di fatto: le imprese multinazionali si sono riunite in un'associazione datoriale (Assodelivery) per evitare la contrattazione e ribadire lo status quo, mentre il Governo è stato del tutto inadeguato a tradurre in legge quanto inizialmente promesso (l'estensione ai riders dei diritti del lavoro subordinato). Se a livello di interventi legislativi non si registra nessun miglioramento, i riders hanno ottenuto i risultati principali nel campo dell'esposizione mediatica - riuscendo a suscitare grande attenzione e partecipazione per la propria lotta.

Un tale interesse e tanto sostegno da parte dell'opinione pubblica non sarebbero però stati possibili senza la capacità dei riders di riuscire ad interpretare una tendenza articolata ben al di là del mondo del food delivery. I riders infatti sono una frazione esigua della forza lavoro (i dati di una ricerca della Fondazione De Benedetti, ripresi dal XVII Rapporto annuale 
dell'Inps ne stimano 10.000 in tutto il Paese) ma le loro condizioni appaiono al centro delle tendenze economiche più recenti. La fuga dalle regole del lavoro subordinato (Srnicek, 2017); la pervasività del controllo digitale, la pressione costante verso un'intensificazione della prestazione (Chicchi, Simone, 2017); il ritorno del cottimo, che invade oggi una vasta area di processi produttivi dalle campagne alle redazioni editoriali, ma anche l'assenza di una copertura da parte dei Ccnl, come accade anche per gli addetti dei call center outbound, sono tutti esempi della valenza generale avuta dalla vertenza dei riders. Non a caso, «Non per noi, ma per tutti» è uno degli slogan che ha accompagnato l'esperienza di Riders Union, segno di una consapevolezza di tale portata.

\section{Riferimenti bibliografici}

Breman J., Van Der Linden M. (2015). Informalizzare l'economia: il ritorno della questione sociale a livello globale. In: Salmieri L., Verrocchio, a cura di. Di condizione precaria. Sguardi trasversali tra genere, lavoro e non lavoro. Trieste: Edizioni Università di Trieste - EUT: 11-32.

Cannavò S. (2018). Mutualismo. Ritorno al futuro per la sinistra. Roma: Alegre.

Cavallini G. (2017). Foodora, Deliveroo \& Co.: le fattispecie della gigeconomy italiana, tra previsioni contrattuali ed effettive modalità di esecuzione del rapporto. Relazione al Convegno internazionale di studio. Impresa, lavoro e non lavoro nell'economia digitale. Brescia, 12-13 ottobre.

Chicchi F., Simone A. (2017. La società della prestazione. Roma: Ediesse.

Comito V. (2018). L'economia digitale, il lavoro e la politica. Roma: Ediesse.

De Stefano V. (2016). The Rise of the "Just in Time» Workforce. Ondemand work, crowdwork and labour protection in the "gigeconomy». Conditions of Work and Employment Series No. 71. Geneva: Ilo -- <https://www.ilo.org/wcmsp5/groups/public/---ed_pr otect/---protrav/---travail/documents/publication/wcms_44326.pdf.>.

Di Vico D. (2017). Nel paese dei disuguali. Noi, i cinesi, la giustizia globale. Milano: Egea

Friedman G. (2014). Workers without employers: Shadow corporations and the rise of the gig economy. Review of Keynesian Economics, 2(2): 171-188. Doi: 10.4337/roke.2014.02.03. 
Marrone M. (2019). Gig economy e sindacalismo informale. In: Somma A., a cura di. Lavoro alla spina, welfare à la carte. Lavoro e previdenza ai tempi della gig economy. Milano: Meltemi.

Prassl J. (2018). Humans as a service. The promise and perils of work in the gig economy. Oxford: Oxford University Press.

Scholz T. (2017). Think outside the boss. L'incapacità di immaginare una vita diversa è il trionfo definitivo del capitale. In: Armano E., Murgia A., Teli M., a cura di. Platform capitalism e confini del lavoro negli spazi digitali. Milano-Udine: Mimesis.

Srnicek N. (2017). Capitalismo digitale. Google, Facebook, Amazon e la nuova economia del web. Roma: Luiss University Press. 